\title{
Cystic Fibrosis in Egyptian Children: Achievements and Future Directions
}

\author{
Mona M. El Falaki, Mona M. El Attar, Nossa El Basha, Nihal El Refai, Walaa Shahin, \\ Hanan Mohsen, Sara Ali, Amr M. Mostafa, Dina H. Hamed* \\ Pediatric Allergy and Pulmonology Unit - Cystic Fibrosis Center. Pediatric Department, Faculty \\ of Medicine, Cairo University, Egypt; monafalaki@hotmail.com, monamohsenm@gmail.com, \\ noussaelbasha3@yahoo.com, nihal-r@hotmail.com,walaashahin@kasraiainy.edu.eg, \\ nana_mohsen2006@yahoo.com,dr.osarahali@gmail.com, amrmustafa366@gmail.com, \\ dina.alkhtaib@kasralainy.edu.eg \\ - Correspondence: dina.alkhtaib@kasralainy.edu.eg \\ Received: 7/12/2020; Accepted: 20/12/202; Published online: 25/12/2020.
}

\begin{abstract}
:
Cystic fibrosis (CF) is the most common potentially lethal and life-shortening genetic disease caused by mutations of the cystic fibrosis transmembrane conductance regulator (CFTR) gene. Clinical consequences of the CFTR defect are site-specific and range from severe (lungs, pancreas, male reproductive tract) to mild (intestine) to asymptomatic (sweat glands). In many developing countries CF has remained largely unrecognized, and inadequately managed, resulting in avoidable death or suffering in infancy, childhood, and adulthood. Delivering adequate $\mathrm{CF}$ services is met by substantial difficulties as the spectrum and distribution of $\mathrm{CF}$ in Egypt are still not well known. Against this background, the aim of this work is to emphasize the challenges facing initiation of a CF center in low privileged settings with the objectives of improving diagnosis, survival, and to eventually provide optimum management. Strategies for the implementation and development of $\mathrm{CF}$ services, as well as proper collection and documentation of patient data are therefore of vital importance. The characterization of the prevalence and molecular genetics of $\mathrm{CF}$ in Egypt is of utmost importance so that appropriate genetic counseling to $\mathrm{CF}$ patients and their families can be achieved and to pave the way for new treatment modalities.
\end{abstract}

Level of Evidence of Study: IV (1).

Keywords: Cystic fibrosis; cystic fibrosis center; challenges; future directions; Egypt

Abbreviations: CF: cystic fibrosis; CFTR: cystic fibrosis transmembrane conductance regulator; CFC-CU: Cystic Fibrosis Center of Cairo University; HFCWO: high frequency chest wall oscillation; NBS: newborn screening programs

\section{Introduction}

Cystic fibrosis (CF) is the most common potentially lethal and life-shortening genetic disease among populations of white Caucasian descent, such as those of Europe, North America and Australasia, being caused by mutations of the cystic fibrosis transmembrane conductance regulator (CFTR) gene (2).

Disruption of CFTR function has distinct consequences for different parts of the body, and certain organs seem to be more sensitive than others. Although CFTR gene expression is found in a number of sites (airway, salivary glands, pancreas, liver, sweat ducts and reproductive tract), it is the impact on the lungs and the gastrointestinal tract that poses the major consequence for morbidity $(3,4)$.

The incidence of $\mathrm{CF}$ varies according to the ethnic origin, ranging from one in 2,000 to one in 3,500 Caucasians born in Europe, the United States, and Canada, and with the lowest incidence among Hispanics (1:8,400 births), African-Americans (1:15,000 births), and the Asian population of Hawaii (1:89,000 births) (5).

For a long time, the exact prevalence of $\mathrm{CF}$ was not known and believed to be non-existing in the Arab world (Northern African countries bordering the Mediterranean and the Middle 
East). The few studies available suspect the presence of many undiagnosed patients and emphasize suspicion of presence of a higher incidence particularly in view of the high consanguinity rate. Furthermore, the population of the region is characterized by a large family size, high fertility rates, high maternal and paternal age and a high rate of marriage among members of the same tribes $(6,7)$. Case reports from several Arabic countries including Saudi Arabia, Bahrain, Tunisia, Algeria, Lebanon and Egypt have suggested estimates ranging from 1 in 2,560 to 1 in 15,876 individuals (8).

Limited data is available regarding CF prevalence among Egyptian. CF has been believed to occur infrequently in Egypt; only few papers suggesting its presence.

In many developing countries $\mathrm{CF}$ has remained largely unrecognized because its clinical features individually resemble respiratory and gastro-intestinal problems associated with malnutrition, failure to thrive and a high infant mortality rate. Without a high index of suspicion and with the belief that $\mathrm{CF}$ does not exist in the population, the diagnosis of CF can be missed. The growing awareness of $\mathrm{CF}$ as well as the increasing availability of diagnostic tests - the sweat test and/or DNA tests - frequently leads to the identification of a higher number of affected individuals (9). Therefore, the aim of this communication is to emphasize the challenges facing initiation of a $\mathrm{CF}$ center in less privileged settings with the objectives of improving diagnosis, survival and to eventually determine the true figure of CF prevalence.

\section{Establishment of The Cystic Fibrosis Center of Faculty of Medicine, Cairo University}

The CF Center of Cairo University (CFC-CU) was established in 2006 by Professor Mona El Falaki, Head of the Pediatric Allergy and Pulmonology Unit in The Children's Hospital, as a specialized center under the umbrella of the Pediatric Allergy and Pulmonology Unit. The CFC$\mathrm{CU}$ is the first referral center of excellence for CF patients in Egypt. It provides diagnosis, management and follow up for $\mathrm{CF}$ patients delivering a multidisciplinary approach through cooperative work between the pediatric pulmonologists and several subspecialties including clinical nutritionists, physiotherapists, radiologists, ear, nose and throat specialists, pharmacists, gastroenterologists, hepatologists, psychologists, genetic reference laboratory physicians and others depending on every patient's specific requirements.

The CFC-CU is also a research and educational center for physicians of different specialties involved in the care of CF patients in Egypt and the Arab world. It stands in the forefront for international cooperation with $\mathrm{CF}$ centers in different parts of the world to exchange expertise, update knowledge and conduct research projects. This cooperation is particularly important with other Arab countries to increase our understanding of the true prevalence of this underdiagnosed condition.

In the period from 2007 to 2019 around 3500 patients were referred for assessment of suspected CF from all over Egypt. Their main clinical presentations were chronic productive cough, repeated pneumonia, failure to thrive, steatorrhea, meconium ileus and rarely hepatobiliary involvement. Age at diagnosis ranged between 8-12 months (mean 9 months +/- 2). At present, 366 patients are included in our registry. Almost $80 \%$ of patients follow up regularly on 2 to 3 monthly basis. To date, the CF cohort suffered 25 deaths that were primarily related to their lung disease.

\section{Difficulties in establishing a CF center in a low privileged setting}

Phenotypic genotypic expression of CF: The CF phenotype is characterized by complex, multiorgan involvement, analyzed in context of its various clinical components such as, pulmonary disease, pancreatic exocrine function or sweat chloride abnormality among others. Clinical consequences of the CFTR defect are site-specific and range from severe (lungs, pancreas, male reproductive tract) to mild (intestine) to asymptomatic (sweat glands) (10). Although cystic fibrosis is a complex disorder affecting many organs, $85 \%$ of the mortality is a result of lung disease (11).

A longitudinal two-year study recruiting 100 patients suspected clinically of having $\mathrm{CF}$ and referred to the CF Center of the Allergy and Pulmonology Unit, Children's Hospital, Faculty of Medicine, Cairo University, Egypt (CFC-CU), reported CF in more than a third of the patients $(36 \%)$. The main clinical presentations in patients referred for sweat chloride testing were: 
chronic cough in $88.9 \%$, failure to thrive in $75 \%$, steatorrhea in $66.7 \%$, and hepatobiliary involvement in $13.9 \%$. The method used for the diagnosis is the sweat chloride test, which is conducted in accordance to the standardized methods approved by the Cystic Fibrosis Foundation (CFF) and the National Committee for Clinical Laboratory Standards guidelines. Positive consanguinity was reported in $50 \%$ of $\mathrm{CF}$ patients (12).

Regarding the prevalence of different CF genetic mutations a preliminary study by El Falaki, 2014, reported the F508 del mutation in $68.8 \%$ of patients, of those $25 \%$ were homozygous and $43.8 \%$ were heterozygous (12). In a more recent study by Shahin et al, 2016, the mutations detected in Egyptian CF patients were F508 del 58 \%, 2183AA/G 10 \%, N1303K 6 \%, R1162X 6\%, A544E 4\%, I148T $4 \%$, W1282X $4 \%$, G155D $2 \%$, CFTRdel2-3 21 KB $2 \%$, 3199del6 $2 \%$ and R347P $2 \%$. There was no association between the initial clinical presentation of CF and the genotypes detected (13).

Nutritional assessment and management of CF is an important aspect of follow up and longterm clinical care. Failure to thrive is a major challenge in Egyptian CF patients. An obstacle in their long-term management is to achieve their nutritional requirements in the form of calories, macronutrients and micronutrients $(14,15)$.

The "gold standard" for diagnosing CF is the sweat chloride test. The method we use is conducted in accordance with the standardized methods approved by the Cystic Fibrosis Foundation (CFF) and the National Committee for Clinical Laboratory Standards guidelines.

Our primary challenge was choosing the technique for sweat collection (following sweat induction by pilocarpine iontophoresis) that would be most suited to our particular conditions and resources. We finally settled on the Macroduct sweat collection system because it is of high sensitivity and specificity, does not need a specialized lab or highly trained personnel to be performed, is less time-consuming and costly and analysis of sweat electrolytes can be performed on even small samples of sweat (16) then the collected sweat is further tested by the colorimetric method for its content of chloride.

The provision of basic and essential medications required for the management of these cases is a continuous struggle in developing countries. CFU-CU has ensured the continued supply of the vital drug which is considered the cornerstone in the management of CF patients and which has still not been included in the essential drug list in Egypt. Due to its unavailability, we have replaced tobramycin (an anti-pseudomonas medication) with other aminoglycosides given by the inhalation route namely gentamicin and amikacin. Other drugs that can be given by inhalation and are available on the Egyptian market include ceftazidime and colistimethate sodium. With regard to inhaled mucolytics and Dornase alfa, they are not obtainable but other inhalation mucolytics as acetyl cystein and hypertonic saline are readily accessible.

CFC-CU is the only center in Egypt equipped with High Frequency Chest Wall Oscillation (HFCWO) and cough-assist machines necessary for performance of chest physiotherapy, which is an integral part of the regular management of this condition. In addition, we teach the families how to perform the traditional manual chest percussion for postural drainage.

\section{Future Directions}

Improving awareness: $\mathrm{CF}$ and CF-related diseases are under-diagnosed in many developing countries due to insufficiency of knowledge in the medical community about the disease as well as poor access to medical facilities and healthcare for CF patients.

Through presentations at meetings, national medical conferences, CF workshops, training courses and sweat testing workshops all over the country, the core members of the CFC-CU are deeply engaged in increasing the awareness of the medical community and primary health caregivers about this condition with the aim of increasing early identification and referral. Clinicians working in remote isolated areas and closed communities, where consanguinity rates are very high, may have difficulties identifying children with atypical presentations and the population distribution of $\mathrm{CF}$ can make access to diagnosis and treatment difficult for a large proportion of affected families. In addition, CF has been included in undergraduate medical school curricula and stressed upon in postgraduate degrees so that future doctors could recognize the condition and know how to deal with it. 
Collaboration between established and emerging CF centers across the country is of utmost importance. This provides regular contact for training, sharing of experiences and pooling of data with the ultimate aim of establishing and maintaining a national CF registry.

Neonatal screening is of particular value in estimating the burden of CF in a population such as Egypt's where the condition is suspected to be underdiagnosed. The first neonatal screening testing aiming at evaluating the magnitude of the CF problem in Egypt was part of a study by Abdel Salam et al. in 1993, using the meconium test strip (BM-mec test), which is the measurement of the albumin level in meconium using a test strip. The prevalence rate was reported to be 1:2664 in 18560 screened newborns and 1:56 in a series of 224 high risk children(17).

There is overwhelming evidence that supports the establishment of newborn screening (NBS) programs for cystic fibrosis to improve the overall survival, long-term growth and health outcomes of people living with CF. NBS most often consists of a two-step screening test using immunoreactive trypsinogen (IRT) from a blood spot procured during the first few days of life that is followed by a search for common mutations in the CFTR gene. A positive CF screen results in a referral to a $\mathrm{CF}$ clinic to confirm the diagnosis through additional blood and sweat testing. Babies with a high risk of having $\mathrm{CF}$ are identified and once the diagnosis is confirmed, education and treatment is initiated, typically in the first 4 to 6 weeks of life (18).

Genetic Epidemiology of CF in Egypt: Knowledge of different mutations shared by regional and other Arab communities is important for the choice of candidate mutations to be included in the diagnostic panel for Egyptian patients. In addition to the routine testing (using commercial genetic assays) carried out in the molecular biology lab, The Children's Hospital, Cairo University, collaborations are ongoing with several genetics laboratories across the world using state-of-the-art techniques for complete sequencing of the CFTR gene.

Through the combination of general carrier screening, neonatal and preconception screening, prenatal diagnosis in addition to confirmation of $\mathrm{CF}$ in patients with typical or atypical presentations or in those with normal or borderline quantitative sweat chloride levels we aim to eventually determine the true figure for cystic fibrosis prevalence in the Egyptian population.

Transition clinics: thanks to the multidisciplinary care in specialized centers the prognosis has improved and CF has become a chronic instead of a deadly disease. Median life expectancy has increased significantly with the increasing number of patients surviving into adulthood. Life expectancy has risen to 40 years or more (19). For this reason, many children require transition to adult $\mathrm{CF}$ care.

Since the establishment of CFC-CU in 2006 many of our patients have reached adulthood. Due to the strong bond created between the patients and members of our team, transition is often met with resistance from the patients and their parents. The age-limit for hospital admission in The Children's Hospital is 12-13 years, although we continue to follow our patients on an outpatient basis for longer than that. When hospital admission is needed by our adolescent patients this takes place in the adult hospital. We always plan for transition from pediatric to adult care to be a gradual process with the $\mathrm{CF}$ patients and their parents being involved at an early stage. Regular communication between the teams during this period is crucial to ensure the success of the transition process.

\section{Conclusion}

$\mathrm{CF}$ and CF-related diseases might be under-diagnosed in Egypt. The spectrum and distribution of $\mathrm{CF}$ are still not well known. Our strategies for the implementation and development of $\mathrm{CF}$ services as well as proper collection and documentation of patient data are therefore of vital importance. The registry that we have begun at CFC-CU will play an essential role in improving our knowledge of CF in Egypt. Characterization of the molecular genetics of $\mathrm{CF}$ in Egypt should be made available so that appropriate genetic services to $\mathrm{CF}$ patients and their families can be achieved. When a suitable mutation panel is set up, CF prevalence in Egyptians can be determined. In addition, understanding genotype-phenotype relations can lead to new targets for treatment and better disease outcome. 


\section{Acknowledgment}

The authors would like to acknowledge the heads, managers and workers in The Department of Clinical and Chemical Pathology, The Pediatric Allergy Unit and Pulmonology including Pulmonary Function Lab, as well as Children's Hospital, Faculty of Medicine, Cairo University management for making this work possible.

\section{Author Contributions:}

M.M.E.F. took the lead in writing the original manuscript draft, conceptualizing, supervising and revising the final manuscript. D. H.H. took charge of the submission process and also contributed to and supervised the requested corrections. M.M.E.A. contributed to and supervised the requested corrections. All authors contributed by data curation, providing critical feedback and revising the final manuscript. All authors have read and agreed to the final version of the manuscript.

\section{FUNDING}

Authors declare there was no extramural funding provided for this work.

\section{CONFLICT OF INTEREST}

The authors have no conflicts of interest related to this work and are responsible for its veracity.

\section{References}

1. S. Tenny, M. Varacallo, Evidence Based Medicine. (StatPearls Publishing; Treasure Island (FL), 2020; https://www.ncbi.nlm.nih.gov/books/NBK470182/).

2. R. Kraemer, D. N. Baldwin, R. A. Ammann, U. Frey, S. Gallati, Progression of pulmonary hyperinflation and trapped gas associated with genetic and environmental factors in children with cystic fibrosis. Respir. Res. 7, 138 (2006).

3. M. Proesmans, Best practices in the treatment of early cystic fibrosis lung disease. Ther. Adv. Respir. Dis. 11, 97-104 (2017).

4. E. Saracević, A. Redzić, Genetic examination of children suffering from cystic fibrosis. Bosn. J. Basic Med. Sci. 5, 69-71 (2005).

5. R. Rodrigues, C. S. Gabetta, K. P. Pedro, F. Valdetaro, M. I. M. Fernandes, P. K. R. Magalhães, J. N. Januário, L. M. Z. Maciel, Cystic fibrosis and neonatal screening. Cad. Saude Publica. 24 Suppl 4, s475-484 (2008).

6. A. S. El-Seedy, H. Shafiek, A. Kitzis, V. Ladevèze, CFTR Gene Mutations in the Egyptian Population: Current and Future Insights for Genetic Screening Strategy. Front. Genet. 8, 37 (2017).

7. D. Al-Sadeq, T. Abunada, R. Dalloul, S. Fahad, S. Taleb, K. Aljassim, F. A. Al Hamed, H. Zayed, Spectrum of mutations of cystic fibrosis in the 22 Arab countries: A systematic review. Respirol. Carlton Vic. 24, 127-136 (2019).

8. M. Kambouris, H. Banjar, I. Moggari, H. Nazer, M. Al-Hamed, B. F. Meyer, Identification of novel mutations in Arabs with cystic fibrosis and their impact on the cystic fibrosis transmembrane regulator mutation detection rate in Arab populations. Eur. J. Pediatr. 159, 303-309 (2000).

9. WHO Human Genetics Programme., The Molecular Genetic Epidemiology of Cystic Fibrosis: Report of a Joint Meeting of WHO/ECFTN/ICF(M)A/ECFS, Genoa, Italy, (2002).

10. E. F. Tizzano, M. Buchwald, CFTR expression and organ damage in cystic fibrosis. Ann. Intern. Med. 123, 305-308 (1995).

11. P. A. Flume, K. A. Robinson, B. P. O’Sullivan, J. D. Finder, R. L. Vender, D.-B. WilleyCourand, T. B. White, B. C. Marshall, Clinical Practice Guidelines for Pulmonary Therapies Committee, Cystic fibrosis pulmonary guidelines: airway clearance therapies. Respir. Care. 54, 522-537 (2009).

12. M. M. El-Falaki, W. A. Shahin, N. R. El-Basha, A. A. Ali, D. A. Mehaney, M. M. El-Attar, Profile of cystic fibrosis in a single referral center in Egypt. J. Adv. Res. 5, 563-568 (2014).

13. W. A. Shahin, D. A. Mehaney, M. M. El-Falaki, Mutation spectrum of Egyptian children with cystic fibrosis. SpringerPlus. 5, 686 (2016). 
14. N. El-Koofy, M. El-Mahdy, M. Fathy, M. El Falaki, D. H. El Dine Hamed, Nutritional rehabilitation for children with cystic fibrosis: Single center study. Clin. Nutr. ESPEN. 35, 201-206 (2020).

15. W. A. Shahin, D. H. E. D. Hamed, N. A. M. El enbaby, S. abd-Elatty Sharaf, R. A. K. Mahmoud, M. M. El-Falaki, Vitamin D and its binding protein in children with cystic fibrosis: A single center study. Egypt. Pediatr. Assoc. Gaz. 64, 131-135 (2016).

16. A. C. V. Mattar, E. N. Gomes, F. V. Adde, C. Leone, J. C. Rodrigues, Comparison between classic Gibson and Cooke technique and sweat conductivity test in patients with and without cystic fibrosis. J. Pediatr. (Rio J.). 86, 109-114 (2010).

17. E. Abdel Salam, S. Samuel, M. Awad, A. El-Marsafy, I.E. AbdelMeguid, J. Azmy, Cystic fibrosis in Egyptian children: neonatal screening and high risk groups. JAC. 4, 313-7.

18. D. Y. F. Mak, J. Sykes, A. L. Stephenson, L. C. Lands, The benefits of newborn screening for cystic fibrosis: The Canadian experience. J. Cyst. Fibros. Off. J. Eur. Cyst. Fibros. Soc. 15, 302-308 (2016).

19. R. H. Keogh, R. Szczesniak, D. Taylor-Robinson, D. Bilton, Up-to-date and projected estimates of survival for people with cystic fibrosis using baseline characteristics: A longitudinal study using UK patient registry data. J. Cyst. Fibros. Off. J. Eur. Cyst. Fibros. Soc. 17, 218-227 (2018).

\section{(c) $(1)(8) \Theta$}

